\title{
ON THE MULTI-PREFERENCE APPROACH TO EVALUATING OPPORTUNITIES
}

\author{
Klaus Nehring and Clemens Puppe
}

\section{N DB}

Working Paper Series \#97-07

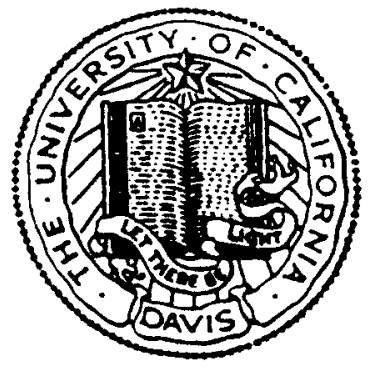

Department of Economics

University of California

Davis, California 95616-8578 


\title{
On the Multi-Preference Approach to Evaluating Opportunities
}

\author{
Klaus Nehring and Clemens Puppe
}

Working Paper Series No. 97-07

February, 1997

Note: The Working Papers of the Department of Economics, University of California, Davis, are preliminary materials circulated to invite discussion and critical comment. These papers may be freely circulated but to protect their tentative character they arc not to be quoted without the permission of the author. 


\section{Introduction}

Imagine an individual who faces the following two-stage decision problem. In the first stage, the individual has to choose among different opportunity sets. In the second stage, exactly one alternative from the set determined by the first stage decision has to be chosen. In such a situation, one may think of two different factors determining first stage choices. First, each menu entails indirect utilaty derived from the ultimately chosen alternative. Secondly, a decision maker might attach intrinsic value to the range of second-stage choices (on the importance of the intrinsic value of choice in individual decision making, see e.g. Jones and Sugden [4] and Sen $[14,15])$. The aim of the present paper is to develop a minimal, and in this sense general theory of "preference for opportunities" that combines both aspects. While perhaps not terribly ambitious, a minimal theory does not seem to be without merits in vew of the conceptual elusiveness and complexity of the notion of "freedom of choice" With our analysis we intend to clarify and lend support to the emerging multi-preference conceptualization of "preference for opportunities" (see e.g Jones and Sugden [4], Pattanaik and $\mathrm{Xu}[10])$. The analysis is minimalistic in that wc consider orderings of sets that. are comparable with respect to set inclusion; such orderings will be referred to as qualitative set orders (QSOs)' 'The proposed theory maintains the least controversial assumption in the context of ranking opportunities, namely that for any given opportunity set no subset can have greater value than the original set (condition M. cf. Sect 2)." The focus is therefore whether or not, for any given pair $(A, B)$ of sets. $A \backslash B$ is of marginal value when $B$ is available. $A$ decision maker's QSO thus describes the value of additional opportunities while being silent about trade-offs.

Given an ordering of the hasic alternatives, the indirect utility principle compares opportunity sets solely on the basis of preference between best elements in each opportunity set $A$ first step beyond this is to assume a "preference for flexibility" due to uncertanty about future tastes (see the classic article by Kreps [5]) For instance, suppose that an individual is uncertain about his preferences between the alternatives $x$ and $y$. Then. in terms of flexibility the set $\{r, \mathrm{y}\}$ would he strictly prefered to either $\{r$ )and $\{y\}$. In general, one would have $A \cup\{x\} \succ A$ if and only if $x$ is superior to all elements in $A$ with posituve probabulaty Intuinvely, preference for flexibility may thus be conceptualized by the notion of expected indirect utility However, this interpretation is unnecessarily particular More generally, if is conceivable that $A \cup\{x\} \succ A$ if and only if $\mathbf{r}$ is superior to all elements in $A$ with respect to some possible preference In contrast to the set of probable preferences, the set of (relevantly) "possible"

"The term "qualitative" refers to the fact that a $Q S O)$ ss only drefined on the domain of all pars $(A, B)$ such that $A \subseteq B$,or $B \subseteq A$

${ }^{2}$ Hence, the theory abstracts from phenomena such as "weakness of will," or .'effort-ofdecision costs," or any other restmitions on the derisin maker's ability to choose from his opportunities 
preferences in general might include any legitimate, or reasonable, or plausible preference ordering even if it has zero subjective probability. In particular, a preference may be possible because it can itself be chosen.

Any of these interpretations entails the following restriction on a QSO. Suppose that for sorne set $A$ the addition of the alternative $x$ is of positive marginal value. Then the addition of $x$ to any subset $\mathrm{B} \subseteq \mathrm{A}$ must be of positive marginal value as well. We will refer to this property as a condition of "Contraction Consistency" (condition C. . cf. Sect. 2) - his condition makes intuitive sense even from a more general perspective based on the notion of diversity of opportunitzes Indeed, suppose that diversity strictly increases when the alternative $x$ is added to the set $\boldsymbol{A}$. Now, any diversity possessed by a subset of $\mathrm{A}$ is also possessed by A Hence, the addition of $x$ to any subset $\mathrm{B} \subset A$ must strictly increase diversity as well. Thus, condition $(C$ seems to be an appropriate general condition for evaluating opportunities. In particular, in the above interpretations in terms of multiple preferences one may allow for co-actualaty of the different preferences. e.g as different "ponts of view." For instance one may derive utility from consumng the fantasy of rloing a multitude of things while knowing that they wor't be done:

By a result of $K r e p s[5]$ conditions $M$ and $C \mathrm{C}$ arc the only restrictions on "preference for flexibility." and hence on "preference for opportunities." Consequently, conditions $M$ anti (' ('art the key axioms of this paper, and our goal is to explore the resulting structure. A QSO satisfying $M$ and $C($ will be refered to as a conststent qualitatue set order (CQSO). Kreps [5] has shown further that II and (C'remain the only restrictions if one assumes an additive, i.e an expected indirect utility representatıon Thus even under that much more structure, the (QSO captures "where the action is."

In contrast to the recent literature on multi-preference conceptualizations of "preference for opportunities" (see [4], [10]). such a conceptualization arises here from a representation theorem. This has two important implications. First, the principle that adding opportunities is always strictly preferred, as sometimes assumed in the literature on freedom of choice." cannot be considered a general principle of evaluating opportunities Secondly. for a typical CQSO the marginal value of adding an alternatue is context-dependent, i.e. in general there exist sets $A . B$ and alternatives $x, y$ such that,

$$
\begin{array}{lll}
B \cup & \text { and } & A \cup\{y\} \sim A, \\
B \cup\{x\} \sim B & \text { and } & B \cup\{y\}>B
\end{array}
$$

This observation suggests that conditions of context independence popular in

'We note that given condition .11 from abowe and transuturity, condition $C C$ is equivalent to Kreps' condition $(15)$ (see [5. 1 ) 567 )

Af. g the principle of stract monotonicity with respect to set inclusion is assumed in (iravel [3] and implied in Pattanaik and Xu [9] Similarly. stret monotonicity is implied in the models considered on Bonsers, Pattanaik and $X_{u}[2]$ 
the literature on ranking sets may not be very helpful in the context of ranking opportunities, see Section 2 for further discussion.

The present paper provides refinements of Kreps' result based on two mot. vations. First. one may want to incorporate (resp. axiomatically characterize) constraints on the set of possible preferences such as the "rigid" superiority of some alternative $\mathrm{x}$ to another $\mathrm{y}$ for all possible preferences. While the implications of rigid weak preference on the CQSO are straightforward, the implications of rigid struct preference are more complex and involve restrictions on the entire CQSO. We are also interested in clarifying the relation between multi-preference representations in an opportunity context and multi-preference rationalization of choice functions as provided by the well-known theorem of Aizerman and Malishevski [1]. Taking a cue from the analysis of Puppe [12], it turns out that on the class of CQSOs satisf ing a condition llE (for. "Irrelevance of Inessential Elements") Kreps representation theorem specializes to that of Aizerman/Malishevski (see Section 7). This raises the question whether IIt is a mere technical artefact or whether it has substance, and more specifically whether IIF can be understood within the multi-preference aproach itself Wo answer this question by characterizing IIE in terms of two alternative conditions on the representation that there exist a representing set of lincar orderings ('Theorem 61 ), or that there exist a set of representing preference orderings that is convex in an appropriate sense (Theorem j) Intriguingly, each of these requrements can be imposed on the multi-preference rationalization of a choice function without loss of generalaty." The notion of convexity, introduced In Vehring [7] as "closedness under compromise" expresses the intuitive idea that orderings that lie "In between" possible orderings should itself be possible This seems a natural enough requirement if "possible" is interpreted as "reasonable," or "legitimate," but less so under a flexibility interpretation of "possible" as "probable" It is not entirely clear how to assess the strength of IIE. While? the linearity characterization imports sornc flavour of genericity on it, we show by means of two examples that it may be rich in implications not obtainable without 11

The paper is organized as follows Section 2 introduces sornc basic definitions and briefly discusses thr sssue of context-dependence Based on the result of Kreps [5] Section 3 derives the representation of a CQSO by means of multi ple preferences on the set of alternatives The interrelation between a CQSO and preferences over alternatives, specifically the problem of rigidity of strict preference. is discussed in Section 4. Section 5 provides the characterization of IIE rankings as those that admit a convex representation. Condition IIE is further examined in Section 6. where it is shown to 1 )? the necessary and sufficient condition lor thr existence of a representation with multiple linear preferences Also it is demonstrated by means of two examples that IIE allows for inference. from partial knouledge of a CQSO As a further application of IIE, Section 7

As to "linearity" this is obvious; as to convexity, see [Nehring 7, Th, 6] 
establishes the link between QSOs and choice functions Concluding remarks arc offered in Section 8 All proofs are found in an appendix

\section{Basic Definitions and Facts}

Let $X$ be a finite set of alternatives and denote by $\mathrm{P}^{0}(\mathrm{X}):=P(X) \backslash\{\emptyset\}$ the set of all non-empty subsets of $X$. By $\Sigma(X)$ we denote the set of all pairs $(\mathrm{A}, B) \in \mathrm{P}^{0}(\mathrm{X}) \times \mathrm{P}^{0}(\mathrm{X})$ which are ordered by set inclusion, i e.

$$
\mathcal{Z}(X):=\left\{(A, B) \in P^{0}(X) \times P^{0}(X): \mathrm{A} \subseteq \mathrm{B} \text { or } \mathrm{B} \subseteq A\right)
$$

By $\succeq$ we denote a reflexive binary relation in $\Sigma(X)$. We call $\succeq$ an ordering in $\Sigma(X)$ if and only if $\succeq$ is complete and transitive in $\Sigma(X)$, ie if anti only if for all $(A, B) \in \Sigma(X),[A \succeq B$ or $B \succeq A]$, and for all $(\therefore, B),(B, C),(A, C) \in$ $\Sigma(X),[A \succeq B$ and $B \succeq C] \Rightarrow A \succeq \bar{C}$ An ordering in $\Sigma(X)$ is also referred to as a qualatative set order (QSO) The intended interpretation of $\succeq$ is that $A \succeq B$ if and only if $A$ entails at least its much "opportunity value" as $B$. The symmetric and asymmetric parts of $\succeq$ are defined as usual. i.e $A-B: \Leftrightarrow[A \succeq$ $B$ and $B \succeq A]$, and $A \succ B: \Leftrightarrow[A \succeq 11$ and not. $B \succeq A]$, respectively Note that by transituity of $\succ$, both relations, and $\succ$, are transitive in $\Sigma(X)$

The intended interpretation of the QSO $\succ$ as describing a "preference for opportunities" is formally captured by the following two basic conditions.

M (Monotonicity) For all B $\subseteq$.I, $A \succeq B$

Monotonicity states that any set $A$ entails at least as much opportunity value as any of 1 ts subsets Note that, given condition $\mathrm{M}$, a binary relation $\succeq$ in $\Sigma(X)$ is automatically complete in $\Sigma(X)$ Furthermore, in this case $\succ$ is transitive In $\Sigma(X)$ if and only if for all sets $A . B . C \in P^{(j)}(X)$ such that $A \subseteq B \subseteq C$. $\left[.4^{-} B\right.$ and $\left.B^{-} C\right] \Leftrightarrow A^{-} C$ The second basic condition is as follows.

$\mathrm{CC}$ (Contraction Consistency) For all $B \subseteq A$ and all $x \in X$.

$$
1 U\{s\} \succ A \Rightarrow B \cup\{\mathbf{s}\} \succ B
$$

Contraction consistency states that if joung the element $x$ to $A$ Increases the entailed opportunity value then this value must also increase when joining $x$ to the smaller set $\mathrm{B} \subseteq A$ Note that since $\succeq$ is reflexive, the element $x \in X \mathrm{in}$ (C cannot be contained in $A$ We will say that an element $r \notin A$ is essentral at $A$ if and only if $A \cup\{x\} \succ A$, that is if and only if it marginally entances opportunty value (Otherwise, if $\mathbf{r} \notin A$ and $A-A \cup\{x\}$ we will say that $x$ is inessential at $A$ Hence, $C$ may be rephrased as follows Suppose that $x \notin A$ is essential at $A$ Then $s$ must be essential at any subset $B$ of $A$

In our approach. we takt conditions $\mathrm{M}$ and $\mathrm{CC}$ as implicitly defining the notion of "preference for opportunitles" Hence. the object of our study is the set of $Q S O S \succeq \operatorname{m} \sum(X)$ which satisfy XI and ( $C$ We refer to it QSO satisfying 
$\mathrm{M}$ and $\mathrm{CC}$ as a conststent qualitative set order (CQSO) and denote the set of all CQSOs in $\Sigma(X)$ by $\mathcal{R}_{C} C(X)$

The simplest examples of CQSOs are indirect utility preferences, A CQSO $\succeq$ is said to be an inderert utility preference (henceforth: Ill-preference) if and only if there exists a complete preference ordering $\mathrm{R}$ on $X$ such that for all $(A, B) \in \Sigma(X)$,

$$
A \succeq B \Leftrightarrow \text { for all } b \in B \text { therc exists } a \in \mathrm{A} \text { such that } a R b
$$

If $\succeq$ is an $I U$-preference with underlying preference ordering $R$, we will write $\succeq=I U(R)$

Any CQSO $\succeq$ canonically induces the following partial order ${ }^{6} R_{\succ}$ on $X$ For all $x, \mathrm{y} \in X^{\prime}$,

$$
د R_{\succ} y \Leftrightarrow\{x\} \sim\{x, y\}
$$

Hence. $x R_{\succ} y$ if and only if $y$ is inessential at $\{x\}$. Note that if $\succeq \in \mathcal{R}_{C C}(X)$ is an $I U$-preference then $-=W\left(R_{\succ}\right)$. The partial order $R_{\succ}$ can be interpreted as the decision maker's contest-independent preference judgements among incremental alternatives added to given opportunity sets. Such an interpretation is indeed warranted due to the following fact.

Fact 2.1 Let $\succeq \in \mathcal{R}_{C}(X)$ and let $R_{\succeq}$ be thr znduced partial order on .Y. Then, $x R_{\geq} y$ if and only if for all $B$ (: such that $13,\{x, y\} \subseteq C$.

$$
B \cup\{y\} \succeq C \Rightarrow B \cup\{x\} \succeq C \text {. }
$$

Hence, $x R_{\succ} y$ if and only if a decision maker would always be willing to exchange $y$ for $x$ independently of the context in which $x$ arid $y$ occur. Note that. in general, for given elements $z, u \in X .(2.2)$ will be true for some $B, C \in P^{0}(X)$ and for others not

If,'-preferences can be characterized on $\Sigma(X)$ by a condition which in effect says that all preference judgements among incremental alternatives are context independent (in the sense? of Fact 2.1).

Theorem 2.1 Let $\succeq \in R_{C C}(X)$. Then $\succeq$ is an $I U$-preference if and only if the induced partial order $R_{\mp}$ is complete on $X$

\footnotetext{
${ }^{6}$ The term "partial order" is sometumes reserved for binary relations that are reflexive. transitive and antisymmetro, whereas a binary relation satisfying just reflexivity and transitivity is sometimes called a prenrder In this paper. antisymmetry is nowhere assumed and both terms are used synonymoush

7'The context-independence condition (2 2) is strong in that it allows tho sets $B$ and $\{x, y\}$ to have non-empty intersection. However. I hr importance of this feature seems rather limited. In particular, in Nehring and Puppe [8] 11 is shown that on non-finite domains even very weak context-independence conditions (with a disjointness-clause) imply thr indirect utility principle provided that the set of alternatives is rich enough.
} 
In concluding this section, we note that in our context the requirement of stract monotonicity with respect to set inclusion would imply $R_{\succeq}=\{(\mathrm{x}, \mathrm{x}): \mathrm{x} \in X\}$ Hence, such a requirement is incompatible with the notion that the decision maker may have some (non-trivial) preferences (s)he is committed to (cf. Son [15], Puppe $[11,12])$

\section{Setting the Stage: The Basic Representation Theorem}

In the following, it will be convenient to work with the asymmetric part $\succ$ of an ordering $\succ$ in $\Sigma(X)$ as the primitive notion. Suppose that. $\succ$ is complete in $\Sigma(X)$, as is e.g. the case if $\succ$ satisfies condition $M$. Then, $A \succ B \Leftrightarrow \operatorname{not} B \succeq A$ Hence, $\succeq$ is transitive if and only if $\succ$ is negatively transitive in the sense that for all $(A, B),(B, C),(A, C) \in \mathrm{Z}(X)$,

$$
[\operatorname{not}(B \succ A) \text { and not }(C \succ B)] \Rightarrow \operatorname{not}(C \succ A)
$$

Let $P_{C}(X)$ denote the set of all asymmetric CQSOs, i.e the set of all relations $\succ$ in $\Sigma(X)$ which are negatively transitive in $\Sigma(X)$ and satisfy conditions $Y$ and ( $\left(C\right.$. Obviously, $>\quad P_{C}(X)$ if and only if tes complement is in $\mathcal{R}_{C C}(X)$

The bask construction of the following analysis leans heavily on Kreps [5] (Our presentation however emphasizes how the multi-preference representation emerges naturally from an analysis of the structure of the set of CQSOS The following fact is easily stablished.

Fact 3.1 The set $P_{(c \cdot C}(X)$ is closed under unouns, l.e. $\succ, \succ^{\prime} \in \mathcal{P}_{C C}(X)$ imples that $\succ U \succ^{\prime} \in \mathcal{P}_{C C}(X)$.

Fact 3.1 suggests the following two questions. What are the CQSOs that are minimal with respect to set inclusion, and can every CQSO he represented as the union of such minmal (CQSOs'. The (non-trivial) minimal (QQSOs are easily characterized. For any $A \in P^{\prime \prime}(X)$ define an clement $\succ_{A}$ of $P_{C C}(X)$ as follows For all $\left(C^{\circ} . D\right) \in \mathcal{Y}\left(X^{\prime}\right)$

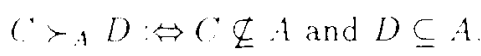

Note that $\succ x=\emptyset$ (Observe 1 lso that. for each $A \in P^{\circ}(X)$. the relation $\succ_{A}$ is the $I U$-preference deriwed from the following preference ordering $P_{4}$ on $X$. For all $x, y \in X$.

$$
x \mathrm{f}^{2}{ }_{A} y \Leftrightarrow \mathrm{x} \notin A \text { and } y \in A
$$

Indeed, it is easily verified that $C \succ A D$ if and only if there exists $x \in C$ such that $x P_{A} y$ for all $y \in D$ ) Hence, $\succ_{A}=L U\left(P_{A}\right)$ In the following, we will refer to the orderings $P_{A}$ and $\succ_{A}$ as dachotomous orderings. Denote by $P_{C r}^{*}(X)$ the set of all dichotomous orderings $\succ_{A}$, i.e

$$
P^{*}(X)=\left\{\gamma A d \in P^{(1)}(X)\right\}
$$


Fact 3.2 The set $P_{C}^{*}\left(X^{\prime}\right) \backslash\{\emptyset\}$ consists rractly of those elements in $\mathcal{P}_{C C}(X) \backslash$ $\{0\}$ that are minimal with respect to set zncluszon

Theorem 3.1 The set $\mathcal{P}_{C C}^{*}\left(X^{*}\right)$ is a base of $\mathcal{P}_{C C}(X)$ in the sense that each element of $\mathcal{P}_{C C}(X)$ as the unon of elements of $\mathcal{P}_{C C}^{*}(X)$. Thut is, for all $\succ \epsilon$ $\operatorname{PCC}_{C}(X)$

$$
>=\bigcup_{A \in \mathcal{A}} \succ_{A} \text { for some family } A \subseteq P^{0}(X)
$$

The proof of 'Theorem 31 uses, for each given $\mathrm{CQSO} \succ$, thr following particular family $\mathrm{A} \subseteq P^{0}(X)$ Let $\succ \in \mathcal{P}_{C C}(X)$, and define a mapping $\mathrm{f} \quad P^{0}(X) \rightarrow$ $P^{0}(X)$ by

$$
f(A):=\bigcup_{\{\in\{A \geq C\}} C
$$

It can be shown that the family $\mathcal{A}=\left\{f(A): A \in P^{0}(X)\right\}$ indeed provides the desired decomposition as stated in theorem 3.1 (for a rigorous proof, see the appendix).

It has already been noted that all relations used for the decomposition of the ordering $\succ$ in Theorem 31 art $11^{\prime}$-preferences. Therefore, one may restate Theorem 3.1 in the following way $A$ relation $\succ$ is in $P_{C C}(X)$ if and only if Ihere exists a finite set $\left\{P_{1}, P_{n}\right\}$ of preference orderings on $X$ such that for all $(A B) \in Z(X)$

$$
\therefore>B \Leftrightarrow \text { for some } 2, A \succ_{1} B
$$

where for each $i \in\{1, \ldots, n\}, \succ_{i}=\mathbb{L}\left(P_{i}\right)$. The sufficiency part of this statement is precisely the content of Theorem 3.1. For the necessity part, note that any $I C$-preference satisfies $I 1$ and $\left(C\right.$, and hence is an element of $\mathcal{P}_{C C}(X)$. By Fact 3.1. $P_{c c}(X)$ is closed under unions, hence any finite union of $I U$-preferences also satisfies $\mathrm{M}$ and $\mathrm{C} C$. Henceforth. we will refer to a set of preference orderings $\left\{P_{1}, \ldots, P_{n}\right\}$ satisfying (3.3) as a representing family of the (QSO $\succ$

In Theorem 3.1. one may think of the elements of the family $\mathcal{A}$ as corresponding to different states. For instance in the specific interpretation adopted in [5] thr elements $\succ_{11 A}$, or rather the corresponding preference orderings $P_{i}$ as at)ove. correspond to different future "tastes" in mutually exclusive states of the world about which an individual is uncertain. In the more general perspective of this paper, the orderings $P_{1}$ may be interpreted as the different viewpoints from which an individual evaluates the elements of $X$. In our framework. the content of Theorem 3.1 may thus he described as follows. If a ranking $\succ$ in $\Sigma\left(X^{\prime}\right)$ exhibits a "preference for opportunities" in the sense of conditions $M$ and CC then there exists a set of viewpoints such that, for all $B \subseteq A, A>B$ if and only if from some virwpoint $A$ is strictly better than $B$. In particular, by Theorem 3.1 one obtains that $x$ is essential at $A$ iff $x$ is uniquely best in $A \cup\{x\}$ from some viewpint $P$ ? 
It can easily be checked that the "state space" (i e the family $A$ in Theorem 31 , or equivalently, a representing family $\left.\left\{P_{1}, \ldots, P_{n}\right\}\right)$ is not uniquely determined by $\succ$ However, as already observed in [5], there are state spaces which deserve special interest. Consider the set $\left\{f(A): A \in P^{0}(X)\right\}$ where f : $P^{0}(X) \rightarrow P^{0}(X)$ is defined as in (3.2) above. A subset $\mathcal{C}$ of this set, is a chazn if and only if $\mathcal{C}$ is completely ordered by set inclusion. A chain $\mathcal{C}$ is marimal if and only if $\mathcal{C}$ is not it proper subset of any other chain Denote by $\Gamma_{\max }$ the set of maximal chains in $\left\{f(A): A \in P^{0}(X)\right\}$. Obviously, cvery $/(A)$ is contained in some maximal chain Hence, the representation in Theorem 31 may be written as,

$$
\succ=\bigcup_{C \in \Gamma_{m a x}}\left[\bigcup_{f(A) \in \mathcal{C}} \succ_{f(A)}\right]
$$

We will refer to this representation of $\succ$ as the marmat chain representation. Observe that since $\mathcal{C}$ is a chain, ach of the relations $\bigcup\left\{\succ_{f 1 A}: f(A) \in \mathcal{C}\right\}$ in (3.4) is an $l U^{\prime}$-preference. Hence, one may think of each maxmal chain as corresponding to one single state. In particular, if $\succ$ itself is already an $l U$ preference then the maximal chain representation of $\succ$ involves only one state.

Using the duality between a $C Q S O \succeq \in \mathcal{R}_{C O}(X)$ and the corresponding strict $C Q S O \succ \in P_{C}(X)$, one may restate Theorem 3.1 in the following way. For each $A \in P^{0}(X)$, let $\succeq_{A}$ denote the weak dichotomous preference corresponding to the ordering $\succ_{A}$ defined in (3 1 ) Furthermore, denote by $\mathcal{R}_{C}^{*}(X)$ the set of all weak dichotomous preferences, ie

$$
R_{(X)}^{*}(X):=\left\{\succeq_{A}: A \in P^{(0)}(X)\right\}
$$

Theorem 3.1' The set $\mathcal{R}_{C}^{*}(X)$ is a ("dual") base of $\mathcal{R}_{\text {Co }}(X)$ in the sense that each element of $\mathcal{R}_{C C}(X)$ is the intersection of clements of $\mathcal{R}_{C C}(X)$. That is, for all $\succeq \in \mathcal{R}_{C C}(X)$.

$$
\succeq=\bigcap_{A \in \mathcal{A}} \succeq \text { f for som famely } A \subseteq P^{(n)}(X)
$$

Clearly, as in the proof of 'Theorem 31 . in order to verify Theorem 31 ' one may use the family $\mathrm{A}=\left\{f(A) \quad A \in P^{0}(X)\right\} \quad$ By 'Theorems 3.1 and $: 31^{\prime}$, It is just a inatter of convenience whether one represents a $C Q S O$ as the intersection of a set of weak $I U$-preferences. or its struct part by the unon of the corresponding strict orderings. In particular, we will also refer to a set $\left\{R_{1}, R_{n}\right\}$ of wrak orderings on $X$ as a representing family for $\succ \in R_{(i,} \cdot(X)$ whenever the set $\left\{P_{1}, \ldots P_{n}\right\}$ of the corresponding strict orderings is a representung family far the corresponding strict ordering $\succ \in F_{r}$, (.Y) in the sense defined previously 


\section{Rigid Preferences over Alternatives}

Let $\succeq \in \mathcal{R}_{C C}(X)$, and let $R_{\succ}$ be the induced partial order on $\mathrm{X}$ defined by (2.1). As we have argued, $R_{\succ}$ may be interpreted as describing a decision maker's context independent preference jugdements involved in the ranking of opportunity sets. The following fact is easily verified.

Fact 4.1 Let $\succeq \in \mathcal{R}_{C C}(X)$ and let $\left\{R_{1}, \ldots, R_{n}\right\}$ be a representıng family of $\succeq$ according to Theorem 3.1'. Then, for all $\mathrm{x}, y \in \mathrm{X}$,

$$
x R_{\succeq} y \Leftrightarrow \text { for all } i \in\{1, \ldots, n\}, x R_{i} y
$$

By Fact 4.1, a weak preference for $\mathrm{x}$ over $y$ is context-independent if and only if it is respected by all viewpoints, or in other words, if and only if it is respected in every possible "preference world" In accordance with terminology in thetheory of possible worlds one might thus call $\mathrm{ft}+$ also the decision maker -; rigut preferences among the clrments of $X$

Also, consider the case where there is an independently given partial order $R$ on $X$ representing the decision maker's (partial) preference judgements on the set $X$. Assume that the ordering $>-$ of sets respects $R$ in the sense that $x R y \Rightarrow$ $x R_{\succ} y$ Then by Fact $1.1, R$ is respected by all viewpoints, I $x R y \Rightarrow x R_{i} y$ for a $i \in\{1 \ldots n\}$ Obviously, analoguous statements are true for the symmetric part $I_{\succ}$ of $R_{\succ}$

A natural question to ask in this context is therefore whether the same applies also to the asymmetrıc part $P_{\succ}$ of $R_{\succ}$ which is defined by

$$
x P_{\succeq} y \Leftrightarrow[\{x\} \sim\{x, y\} \text { and }\{x, y\} \succ\{y\}] .
$$

for all $\mathrm{x}, y \in X$ Perhaps surprisingly, the answer is no To see this, consider the following example

Example 4.1 Let $X=\{x \quad y z\}$ and define an element $\succeq \in R_{C O}(X)$ as follows For all $(A, B) \in \Sigma(X)$

$$
A \succeq B \Leftrightarrow[A=B \text { or }(\mathrm{A} \neq\{\mathrm{y}\} \text { and } \mathrm{A} \neq\{z\})]
$$

It can be verified that for instance. $\left\{R_{1}, R_{2}\right\}$ with

$$
x I_{1} y P_{1} z \text { and } x I_{2} z P_{2} y
$$

Is a representing family of weak orders for $\succ$ Note that for the ordering $\succ$ defined above one has $x P_{\succ} y$ However, the weak order $R_{1}$ does not respect this strict preference judgement. Indeed, in this example there cannot exist a representing family $\left\{R_{1}, \quad\right.$. R,, $\}$ such that each $R_{2}$ respects the strict preference for $x$ over $y$ in the sense that for all i, $x P_{i} y$. To see this, assume that for each z, $x P_{i} y$ First, observe that since $\{y, z\} \succ\{z\}$ there must exist $\jmath \in\{1, n\}$ such that $y P_{j} z$ Hence by transitivity one could conclude $x P_{j} z$ and $x P_{j} ! y$ which 
would imply $\{\mathrm{x}, \mathrm{y}, \hat{z}\}>\{\mathrm{y}, z\}$ However, this is false by assumption

By this example, the induced stricl preference relation $P_{\rightarrow}$ cannot always be interpreted as a rigid strict preference In the following, we will characterize the class of orderings in $\mathcal{R}_{C C}(X)$ for which an Interpretation of $P_{\succeq}$ as the rigid strict preference judgements is possible The characterization is based on the following condition of strict monotonucity Let $Q$ be a binary relation on $X$ Say that $\succeq$ s strictly monotone with respect to $Q$ if and only if $\succeq$ satisfies the following condition

$\operatorname{SM}(Q)$ (Strict Monotonicity) For all $\mathrm{A} \in P^{0}(X)$, and all $\mathrm{x}, \mathrm{y} \in X$ with $x Q y$,

$$
A \cup\{y\} \succ A \Rightarrow A U\{y\} U\{T) \succ A U\{y\}
$$

Intuitively this condition rnay be paraphrased as follows. Suppose that $x Q \eta$. le suppose that $x$ is " $Q$-preferred" to y. Condition $\operatorname{SM}(Q)$ states that, if adding $\mathrm{y}$ is of value, then adding $\mathrm{x}$, which is Q-preferred to $\mathrm{y}$, must be of even greater value The following theorem shows that $S M(P>)$ is the necessary. and sufficiont condition for $P_{\succ}$ being interpretable as the decision maker's rigid struct preference judgements In the theorem. it is conventent to work with the asymmetric part $\succ$ of an ordering $\succeq \in \mathcal{R}_{C C}(X)$

Theorem 4.1 let $>\in \mathcal{F}_{C C}(X)$. There exists a representung famaly $\left\{P_{1} . \ldots, P_{n}\right\}$ such that

$$
x P_{\rightarrow}^{P} y \Leftrightarrow \text { for alli }\{1, n\}, x P_{i} y
$$

If and only if $\succ$ is strictly monotone with respect to $P_{\succ}$, i.e. if and only if ithe complement $\succeq$ of) $>$ satisfies condition $S M\left(P_{\geq}\right)$.

Consider now the cast: where in addition to the ranking $\succeq m \Sigma(X)$ there is an independently given partial preference relation $P$ on $X$ which is asymmetric and transitive Furthermore suppose that $\succeq$ respects $\mathrm{P}$ in the sense that for all $x, y \in X$

$$
x P y \Rightarrow x P>y
$$

The followng result, which is proved along the same lines as Theorem 4.1 gives the necessary and sufficient condition under which $P$ can be interpreted as the decision maker's rigid strict preferences

Corollary 4.1 Let $\succeq \in \mathcal{R}_{C O}(X)$, and let $P$ be a strict partual order on $X$. There exists a representing famtly $\left\{R_{1}, \ldots, R_{n}\right\}$ such that for all $i \in\{1, \ldots, n\}$. $x P y \Rightarrow x P_{1} y$ if and only if $:$ satisfies (4.1) and condition $S: M(P)$. l.e. struct monotonacty wath respect to $\bar{P}$.

\section{Closedness under Compromise}

Any family $\left\{R_{1}, \quad, R_{n}\right\}$ of preference orderings on $X$ can be represented It $>$ i family $u=\left\{, \quad u_{n}\right\}$ of utility functions on $X$ in the sense that for all 
$\imath \in\{1, \quad, \mathrm{n})$ and all $x y \in X$

$$
x R_{i} y \Leftrightarrow u_{i}(x) \geq u_{i}(y)
$$

Say that a family $\left\{R_{1}, R_{n}\right\}$ is closed under compromese if and only if there exısts a family $U$ of representing utility functions that is convex, ie for all $\lambda \in[0,1], u, v \in \mathcal{U}$ implies $X u+(1-\lambda) v \in \mathcal{U}$ A property such as convexity seems to be suitable to distinguish the interpretation of "possible" preference as reasonable, or legitimate preference from the flexibility interpretatıon of "possible" preference as probable preference Indeed, there seems to be good reason to assume that a convex combination of legitimate (or reasonable) preferences should itself be legitimate (reasonable) In contrast, this does not seem to apply to the case of probable future preferences For instance a decision maker may be uncertain about her/his preferences between $x$ and $\mathrm{y}$ while knowing for sure that $(s)$ he will never be indifferent On the other hand if both st rict preferences ${ }_{x} P y$ and $y P x$ are legitimate one would feel that $x I y$ must be legitimate too

Convexity of sets of preferences has ben introduced in Nehring [7] as closed ness under compromise to clarify what it means to rationalize a choice function in terms of a set of preferences While at was shown there that convexity can be required without loss of generality, it adds a surprising amount of structure in the present context For Instance. It will be shown in the next section that it implies rigidity of struct preference. I $e$ it implies condition $S M\left(P_{\succ}\right)$ Moreover. it implies the following property

IIE (Irrelevance of Inessential Elements) For all $A \in P^{0}(X)$ and all $x y \in X$ such that not $x I_{\succ} \mathrm{y}$

$$
[A \cup\{\mathrm{x}\} \mathrm{u}\{\mathrm{y}\} \sim A \mathbf{u}\{\boldsymbol{s}\} \text { and } A \cup\{\mathrm{x}\} \cup\{y\}-\boldsymbol{A} \mathbf{u}\{y\}] \Rightarrow \mathrm{A} \cup\{x y\}-1
$$

The intuition behind IIE is as follows. Suppose that in a set containing $\boldsymbol{s}$ and $y$ the deletion of ether $x$ and $y$ does not reduce the entailed opportunity value. Then the joint deletion of' $x$ and $y$ does not reduce opportunty value cither In this sense, inessential elements arr irrelevant for the ordering $\succeq$ This seems to be plausible enough except in the case where $\boldsymbol{x}$ and $y$ arc indifferent from every relevant vewpont Indeed. suppose that $x$ and y art indifferent In any possible 'preference world.' Ie suppose that $x I_{>} y$ Then the set $A \cup$ $\{\boldsymbol{x}\} \cup\{\mathrm{y}\}$ is indifferent to both $A \mathrm{u}\{\boldsymbol{s}\}$ and $A \cup\{y\}$ However, if from some viewpoint all elements of $A$ are inferior to $s$ and $y$. one would obtain $A \cup\{x, y\} \succ$ $A$. in contradiction to the conclusion of IIE Hence, the clause excluding rigid indifference between $\boldsymbol{x}$ and $\mathrm{y}$ in $\mathrm{ll} \mathrm{E}^{8}$

\footnotetext{
'One way to think about the clause is as follows. For $x \in X$, denote by [x] the equivalence chass of $x$ with respect to the equivalence relation $I_{\succ}$. Then IIF, is equivalent to the following condition. For all $A$ and all $x, y, A \cup\{[x]\} \cup\{[y]\} \sim A \cup\{[x]\}$ and $A \cup\{[x]\} \cup\{[y]\} \sim A \cup\{[y]\}$ implies $A \cup\{[x],\{y]\} \sim A$. Hence, [IE with clause on $X$ is equivalent to [IE, with or without clause, on the quotient space. A condition equivalent to ILE without clause has been introduced in $\{12\}$ where the analysis is implicitly restricted to the quotient space.
} 
Theorem 5.1 Let $\succeq \in \mathcal{R}_{C C}(X)$. There exists a representıng famıly for $\succeq$ that is closed under compromase if and only if $\succeq$ satisfies $I I E$.

As an illustration of Theorem 5.1, consider the CQSO $\succeq$ defined in Example 41 Obviously, $\succeq$ does not satisfy IIE. Indeed, by definition one has $\{x, y, z\}-\{s, z\}$ and $\{x, y, z\} \sim\{y, z\}$, but $\{x, y, z\} \succ\{z\}$ although $x$ and $y$ are not rigidly indifferent. Accordingly, there cannot exist a representing family that is closed under compromise. This can be verified as follows. Let $\left\{R_{1}, \ldots, R_{n}\right\}$ be any representing family for $\succ$. Since $\{x, y, z\}^{-}\{x\}$ and $\{y, z\} \succ\{y)$, there must exist $\mathrm{R}$, such that $x R_{i} z P_{i} y$. Similarly, since $\{x, y, z\} \sim\{x\}$ and $\{y, z\} \succ\{z\}$, there must also exist $R_{j}$ such that $x R_{j} y P_{j} z$. Closedness under compromise would imply the existence of $R_{l}$ such that $x P_{l} y$ and $x P_{l} z$. However. this is not possible since by definition, $\{x, y, z\} \sim\{y, z\}$.

\section{On the Structure of IIE Orderings}

In the "possible preference worlds," ex-post indifference is arguably pointless. or irrelevant. at least unless alternatives are ex-anti. (i.e rigidly) indifferent " Consequently, it seerns natural to require a representing family of a CQSO to effectively consist of linear orderings. Say that a representing family $\left\{R_{1} \ldots, R_{n}\right\}$ of a CQSO is effecturely linear if and only if for all $x, y \in X$.

$$
x l_{\imath} y \text { for some } i \in\{1, \ldots, n\} \Rightarrow x I_{\succeq ! !}
$$

Hence, a representing family is effectively linear if and only if any indifference is rigid. As it turns out, the requirement that any indifference be rigid is equivalent to the requirement of closedness under compromise, and hence to IIL:

Theorem 6.1 Let $\succeq \in R_{C C}(X)$ There exists a representing famaly for $\succeq$ that is effectively linear if and only if $\succeq$ satisfies IIE

Note that Theorems 6.1 and $A 1$ entail that for CQSOs, IIE implies $S M\left(P_{\succ}\right){ }^{10}$ The converse is. however, not true as the following example shows

Example 6.1 Let $X=\{x, y, z\}$ and define a CQSO $\succ \in \mathcal{R}_{C C}(X)$ as follows For all $(A, B) \in \Sigma(X)$.

$$
A \geq B: \Leftrightarrow[A=B \text { or } \# A \geq 2]
$$

Note that for no $x, y \in X, x P_{\succ}^{\prime} y$, hence $\succ$ trivially satisties $\operatorname{SM}\left(P_{\succ}\right)$. Nlso observe that $x I_{\succ} y \Leftrightarrow x=y$. It can be verified that, for instance, the set $\left\{R_{1}, R_{2}, R_{3}\right\}$ with

$$
x I_{1} y P_{1} z, x I_{2} z P_{2} y \text { and } y I_{3} z P_{3} x
$$

\footnotetext{
${ }^{9}$ for an analysis of the role of indifference in the context of freedom and Hexibility supporting this view, see $[12$, Sect. 6$]$

${ }^{10}$ This can, of course, also be shown directly. using $\mathrm{M}$ and $\mathrm{C} C$ :
} 
is a representıng family of weak orders for $\succeq$ Obviously, $\left\{R_{1}, R_{2}, R_{3}\right\}$ is not (effectively) linear. Indeed, there cannot exist a representation with linear ordering since in that case $\{x, y, z\}$ would have to be strictly preferred to one of the sets $\{\mathrm{x}, \mathrm{y}),\{x, z)$, or $\{y, z\}$. However, by the definition of $\succeq$ this is not the case.

It is not entirely clear how strong an assumption IIE really is. While the linearity characterization of Theorem 6.1 suggests the generic applicability of IIE in some sense, the following two examples show that IIE may be rich in implications.

Example 6.2 Denote by $R$ the set of real numbers. Let $X=R^{2}$ and let $\succeq$ be an ordering in $\mathrm{C}\left(\mathrm{R}^{2}\right)$ such that $\succeq$ satisfies $\mathrm{M}$ and $\mathrm{CC}$ Furthermore, suppose it Is known that for all $\mathrm{a}, \mathrm{b}, \boldsymbol{x} \in \mathrm{R}^{2}$,

$$
\{a, b\}-\{a, b, T\} \Leftrightarrow r \in \operatorname{co}\{a, b\},
$$

where co.4 denotes the convex hull of $A$. Then IIE implies the following property. For all $A \in P^{\prime \prime}\left(\mathbf{R}^{2}\right)$,

$$
r \in \cos A \Rightarrow A \sim A \cup\{x\}
$$

In order to verify this claim. suppose that $r \in$ coA 'There are two possible cases

Case 1. There exist two ponts 01' $A$, say $a_{1}$ and $a_{2}$, such that $x \in \operatorname{co}\left\{a_{1}, a_{2}\right\}$. Then, by assumption $\left\{a_{1}, a_{2}\right\}-\left\{a_{1}, a_{2}, x\right\}$, hence by application of CC, $A$ $A \cup\{x\}$.

Case 2. There do not exist two points as in Case I. It is easily verified that in this case there must exist three points of $A$, say $a_{1}, a_{2}$ and $a_{3}$, such that $x \in \operatorname{co}\left\{a_{1}, a_{2}, a_{3}\right\}$ Consider the straight line through $a_{1}$ and $x$, and denote by $y$ the intersection of this line with the line segment $\overline{a_{2} a_{3}}$ as shown in Figure 1

\section{Inserl Figure 1 aboul horc}

By assumption. $\left\{a_{1}, y\right\} \sim\left\{a_{1}, x, y\right\}$ and $\left\{a_{2}, t_{3}\right\} \sim\left\{a_{2}, a_{3}, y\right\}$. This implies by $(C$

$$
\left\{a_{1}, a_{2}, a_{3}, y\right\} \sim\left\{a_{1}, a_{2}, a_{3}, x, y\right\} \text { and }\left\{a_{1}, a_{2}, a_{3}, x\right\} \sim\left\{a_{1}, a_{2}, a_{3}, x, y\right\} \text {. }
$$

respectively From this, one obtains by IIF. $\left\{a_{1}, a_{2}, a_{3}\right\} \sim\left\{a_{1}, a_{2}, a_{3}, x, y\right\}$ which finally implies $A^{-} A \cup\{x\}$ using $M$ and $C C$.

Example 6.3 As in the previous example, let $X=\mathbf{R}^{2}$ and let $\succeq$ he an ordering in $\Sigma\left(\mathbf{R}^{2}\right)$ satisfying $\mathrm{H}$ and $C($ Suppose it is known that

$$
A \sim B \Leftrightarrow A \subseteq \sim \circ B
$$


for all $\mathrm{A}, \mathrm{B} \in P^{0}\left(\mathbf{R}^{2}\right)$ such that $B \subseteq A$ and such that $A$ has at most 4 elements Then, IIE implies that (6.1) holds for all finite sets $A, B \in P^{0}\left(R^{2}\right)$ with $B \subseteq A$ In order to verify this, let $B \subseteq \mathrm{A}$ First, it is shown that $A \subseteq \operatorname{coB}$ implies $B^{-} A$. Let $A=\left\{x_{1}, \ldots, x_{n}\right\} \cup B$, and consider for every $i \in\{1, \ldots, n\}$ the set $B \cup\{x$,$\} . By the argument given in the previous example, one has B-B \cup\left\{x_{i}\right\}$ for every $\mathrm{i} \in\{1, \ldots, \mathrm{n})$. Using $\mathrm{M}$ and $C \mathrm{C}$, this implies by induction $\mathrm{B}-\mathrm{A}$.

Next, let $x \in A \backslash \operatorname{coB}$. By the separating hyperplane theorem, there exists $a$ straight line $l_{1}$ separating the point $x$ and the set $c o B$. Now one can construct two further straight lines $l_{2}$ and $l_{3}$ as shown in Figure 2 such that the set $c o B$ is contained in the triangle spanned by the intersection points $t_{1}, t_{2}$ and $t_{3}$ of these straight lines.

$$
\text { Insert Figure } 2 \text { about here }
$$

By the first part, $\left\{t_{1}, t_{2}, t_{3}\right\} \sim\left\{t_{1}, t_{2}, t_{3}\right\} \cup B$. By assumption. $\left\{t^{2}, t_{1}, t_{2}, t_{3}\right\}>$ $\left\{t_{1}, t_{2}, t_{3}\right\}$, hence using transitivity and $M$,

$$
\left\{x, t_{1}, t_{2}, t_{3}\right\} \cup B>\left\{t_{1}, t_{2}, t_{3}\right\} \cup B
$$

This finally implies by $C C,\{x\} \cup B \succ B$ and therefore $A \succ B$

\section{Multiple Preferences and Choice Functions}

In this section, Theorem 61 is applied in order to uncover a structural isomorphism between the subclass of QSOs satisfying IIE and choice functions. As in Puppe [12], define for each set $A \in P^{0}(X)$ its subset of essential elements $E(A) \subseteq A$ by

$$
E(A):=\{x \in A \quad A \succ A \backslash\{x\}\}
$$

For notational conventence. In (71) we have set $A \succ \emptyset$ for all $A \in P^{0}(X)$ Hence in our terminology, $x \in E(A)$ if and only if $x$ is essential at $A \backslash\{x\}$

Fact 7.1 Let $\succeq$ be an ordering in $\check{U}(X)$ satisfying conditions. $M$ and IIt such that for all $x, y \in X, x I>y \Rightarrow x=y$. Then.

(i) for all $(A, B) \in \Sigma(X), A \succ B \Leftrightarrow(A \backslash B) \cap E(A) \neq 0$.

(ii) for all $A \in P^{0}(X), E(A) \neq \emptyset$.

Consider now an independently given mapping

$$
G: P^{0}(X) \rightarrow P^{0}(X)
$$

such that for all $A \in P^{0}(X), G(A) \subset A$. The interpretation is that the corrspondence $G$ associates to each $A \in P^{(0)}(X)$ the subset of "potentially valuable" alternatives in $A$. Consider the following (novel) condition. 
$\operatorname{SD}(G)$ (Strict $(G$-Dominance) For all $(A, B) \in \Sigma(X)$,

$$
A \succ B \Leftrightarrow(A \backslash B) \cap G(A) \neq \emptyset
$$

Hence, by $\mathrm{SD}$, the set $\mathrm{A}$ is strictly preferred to $\mathrm{B} \subseteq A$ if and only if 1 contains some potentially valuable alternative that is not available in B Condition SD may be viewed as generalizing, at least pruma facie, the account of preference for opportunities offered in Section 3 Let $\left\{P_{1}, \quad, P_{n}\right\}$ be a set of linear orderings and denote, for each $A \in P^{0}(X)$, by $\max _{P_{1}}$ A the (singleton-)set of maximal elements in A with respect to $P_{i}$ If one defines for all $\mathrm{A} \in P^{0}(X)$,

$$
G(A)=\bigcup_{i \in\{1, \ldots, n\}} \max _{P_{i}} A,
$$

condition $\operatorname{SD}(G)$ coincides with (3.3) Other interpretations of $(i$ and $\operatorname{SD}(G)$ include the following ( $(A)$ may describe a set of acceptable alternatives based on the partial elicitation of the decision maker's preferences, and $\operatorname{SI}(G)$ an assessment of flexibility value based on the expectation of further clicitation in the sccond stage of choice. Alternatively, $G(A)$ may represent the set of alternatives that are "normatively acceptable," or "reasonably eligible," and SD(6) a condition reflecting an "intrinsic value of freedom of choice." Interestingly enough, any ordering $\succeq$ derived from some $G$ via SD must satisfy $11 \mathrm{E}$. In particular $\operatorname{SD}(G)$ yields IIE without any assumptions on the choice function

Fact 7.2 Let $\succeq$ be a reflexte and complete binary relateon in $U(X)$. and let $G: P^{0}(X) \rightarrow P^{0}(X)$ be guen such that the asymmetre part $\succ$ of $\succeq$ satusfies condition $S D(G)$. Then.

(i) for all $A \in P^{0}(X)$. $(i(A)=E(A)$.

(ii) $\succ$ is transituve on $\Sigma(X)$.

(iii) for all $x, y \in X, x I_{\succ} y \Leftrightarrow r=y$.

(iv) $\succeq$ satisfies condition .H

(v) $\succeq$ satisfies condition $/ 1 \mathrm{E}$ :

By Fact $72(1)$, condition SD identifies the sets of essential elements with the sets of "potentially valuable" elements. Conversely, by Fact 7.1 any ordering $\succ$ satisfying conditions $\mathrm{II}$ and $\mathrm{IIE}$, satisfies condition $\mathrm{SD}$ with respect to $G=E$ provided that any $I_{\succ}-$-indifference $u s$ trivial

('ondition SI) indeed establishes a structural isomorphism between the subclass of QSOs satisfying IIE and choice functions The following result describes some of the connections between propertics of the ranking $\succ$ and well-known consistency properties of the "choice function" $\left(x \cdot P^{0}(X) \rightarrow P^{0}(X)\right.$ (sef' e g Sen [13], Aizerman and Malishevski [1], Moulin [6])

Theorem 7.1 Let $\succ$ be a complete binary relation in $\Sigma(X)$ such that its asymmetric part $\succ$ satusfies condation $S D$ with respect to $\left(B: P^{\circ}(X) \rightarrow P^{\circ}(X)\right.$. Then $\succ$ satisfies condation (C if and only if $G$ satisfies the following condition. For 
all $A, B \in P^{0}(X)$ with $B \subseteq A$,

(c) $B \cap G(A) \subseteq G(B)$.

Furthermore, if $\succ$ is negatively transituve then $G$ satisfies the follounng so-called "Aizerman" condztzon For all $A, B \in P^{0}(X)$ wzth $B \subseteq A$,

(Aiz) $\quad G(A) \subseteq 13 \Rightarrow G(B) \subseteq G(A)$

Conversely, if $G$ satisfies $(\alpha)$ and (Aiz) then $\succ$ is negatively transatuve

Thus, the key consistency conditions defining a CQSO correspond to the basic rationality conditions on choice functions, $(\alpha)$ arid (Aiz). This nicely confirms the claimed generality of the CQSO approach (see the concluding section for further discussion) Notably absent is "expansion consistency" (Sen's $\gamma$ ), which would translate into the following condition on CQSOs If for all $x \in A$. $\{y, x\} \succ\{x\}$, then $\{y\} \cup A \succ A$ However, the status of this condition as a rationality requirement is less clear. Indeed the appeal of expansion consistency has already been questioned in Aizerman and Malishevski [1] and Nehring [7]

Combining Theorems 7.1 and 6 . I one obtains the following result which has first been proved by Aizerman and Malishevski [I] (see also [6]).

Corollary 7.1 (Aizerman and Malishevski) Let $G \cdot P^{0}(X)-P^{0}(X)$ be a mapping with $G(A) \subseteq A$ for all $A \in P^{0}(X)$. Then $G$ salusfes $(\alpha)$ and $(A 2 z)$ if and only if there exists a set $\left\{P_{1}, \ldots, P_{n}\right\}$ of lenear orderings on $X$ such that for all $A \in P^{\cup}(X)$.

$$
\left(i(A)=\bigcup_{i \in\{i, \ldots, n\}} \max _{P_{i}} A\right.
$$

Note that, conversely. Corollary 7.1 could he used to deduce Theorem 6.1. Indeed, suppose without loss of generality that for all $\mathrm{x}, y \in X, x I_{\succ} \mathrm{y} \Leftrightarrow x=y$, and let $\succ \in P_{C C}(X)$ satisfy IIE By Fact $71 . \succ$ satisifes SD with respect to the correspondence $G=E$ where $E: P^{0}(X)-P^{0}(X)$ is defined as in (7.1). By Theorem 7.1, E satisfies $(\alpha)$ and (Aiz), hence by Corollary 7.1. E can be "rationalized" by a set of linear orderings $\left\{P_{1}, \ldots, P_{n}\right\}$. It is then casily shown that this set $\left\{P_{1}, \ldots, P_{n}\right\}$ constitutes a representing family for the ordering $\succ$. Note. however, that our proof in the appendix entails a somewhat stronger result than Theorem 6.1. In that it shows that given IIE the maximal chain representation of a CQSO is effectively linear: this would also seem to lend support to the genericuty interpretation of the result

\section{Conclusion: On the Generality of CQSOs}

In this paper, we have developed a theory of "preference for opportunities" based on two simple axloms Condition 11 sems to be uncontroversial hence the crucial condition is C ( an C C aspre to the status of a qeneral axiom of "consistent" preference for opportunities?" Quite possibly, as we shall arguc in 
concluding this paper based on the discussion of an apparent counterexample, provided that the "alternatives" are appropriately specified as the carriers of all value. Consider an agent whose choices between behaving "commonly selfishly" $(x)$, "cheaply" (y) and "magnanimously" $(z)$ are described as follows

$$
C(\{x, y\})=\{x\}, C(\{x, z\})=\{x\}, C(\{y, z\})=\{z\} \text { and } C(\{x, y, z\})=\{z\}
$$

The underlying story might be that while the agent is naturally inclined to behave commonly selfishly, slhe is roused to magnanimity in the presence of an opportunity for cheap behaviour. If one distinguishes magnanimity when cheapness is feasible $\left(z_{\ni y}\right)$ from magnanimity when cheapness is not feasible $\left(z_{\not y}\right)$, these choices can be rationalized by the preference ordering $z_{\ni y} P x P z_{z y} P y$ on the set $X=\left\{x, y, z_{\exists y}, z_{\not y}\right\}$ Ranking sets by their chosen element yields the following ordering $\succeq$ on $P^{0}(X)$ with $X=\{s, y, z\}$

$$
\{x, y, z\} \sim\{y, z\}>\{x, z\} \sim\{x, y\} \sim\{x\}>\{z\}>\{y\}
$$

Let $\succeq_{\text {n }}$ denote the restriction of $\succeq$ to $\Sigma(X)$ Obviously, $\succ_{0}$ is transitive in $\Sigma(X)$ and satisfies $M$ (hoice is valued here specifically the possibility of being magnanimous in the face of the opportunty of being cheap in the sense that $\{y, z\} \succ_{0}\{y\}$ as well $a s\{y, z\} \succ_{0}\{z\}$, arid $\{x, y, z\} \succ_{0}\{s, y\}$ as well as $\{r, y, z\} \succ_{0}\{x, z\}$ On the other hand. (C) is violated, since $\{x\} \sim_{0}\{x, z\}$ while $\{\mathrm{x} . \mathrm{y} . \mathrm{z}\} \succ_{\sigma}\{\mathrm{x} . \mathrm{y}\}$ Clearly, if one redescribes sets as subsets of the refined universe $X$. (C (appropriately applied) is sat isfied again.

The above example shows how particular instances of a context-dependence of the value of elements can be accommodated by including the relevant features of the "context" in the specification of an element. Sometimes it is asserted that the process of chotce has intrinsic value itself Jones arid Sugden, for instance. substantiate that intuition by developing an interesting argument for the value of "sagnificant choice" which occurs when a person "while choosing reasonably, acts contrary to a preference that he might reasonably have had" $([4$, p.60]). Notions of the intrunsic value of significant choice and the "process of choice" more generally may ${ }^{11}$ thus lead to pervastve context-dependence. While this would not invalidate $\mathrm{M}$ and $\mathrm{C}$. It would rob these conditions of their bite, at least without additional structure on the nature of the context-dependence.

\section{Appendix: Proofs}

Proof of Fact 2.1 Suppose that $x R_{\succeq} y \in \in\{\alpha\} \sim\{x, y\}$ By CC this implies $B \cup\{\mathrm{x}\}-B \cup\{x y\}$ Furthermore, $\mathrm{M}$ implies $\mathrm{MU}\{x, y\} \succeq B \cup\{\mathrm{y}\}$ Therefore $B \cup\{y\} \succeq C$ implies $B \mathrm{U}\{\mathrm{r} \quad y\} \succeq C$, and hence $B \mathrm{U}\{x\} \succeq C$ Conversely, (2 2) implies $x R_{\succ} y$ by letting $B=\{x\}$ and $C=\{x y\}$

\footnotetext{
11 May, since we do not know of any wroked wut theory articulating these intuitions.
} 
Proof of Theorem 2.1 Clearly, if $\succeq$ is an $I U$-preference the induced partial order $R_{\succ}$ is complete. Conversely, let $R_{\succ}$ be complete on $X$. In order to show $\succeq=I U\left(R_{-}\right)$we have to verify that for all $(\mathrm{A}, \mathrm{B}) \in \Sigma(X)$.

$A \succeq B \mathbf{a}$ for all $\mathrm{b} \in B$ there exists $a \in \mathrm{A}$ such that $a R_{\succ} b$.

If $B \subseteq A,(\mathrm{~A} .1)$ is trivially satisfied. Hence, let $\mathrm{A} \subseteq B$, and let $a^{*}$ he a maximal element in $A=\left\{x_{1}, \ldots, x_{m}\right\}$ with respect to $R_{\succ}$, i.e. $\left\{a^{*}\right\} \sim\left\{a^{*}, x_{i}\right\}$ for all $\mathrm{i}=1, \ldots, m$. First, suppose that $A \succeq \mathrm{B}$. By $\overline{\mathrm{C} C},\left\{a^{*}, x_{i}\right\} \sim\left\{a^{*}, \boldsymbol{x}_{1}, x_{j}\right\}$ for all $\mathrm{i}, j$, hence by transitivity $\left\{\mathrm{a}^{*}\right\} \sim\left\{\mathrm{a}^{*}, x_{i}, x_{j}\right\}$ for all $i, j$. Thus, by induction one obtains $\left\{a^{\prime}\right) \sim \mathrm{A}$, and hence by transitivity, $\left\{a^{\prime}\right\} \succeq \mathrm{B}$. This implies. by condition $M$ and transitivity, $a^{*} R_{\succ} y$ for all $y \in B$. Next, suppose that the righthand side of $(A . l)$ is satisfied. Then, $a^{*} R_{\succeq} \mathrm{y}$ for all $\mathrm{y} \in B$, hence by induction and $\mathrm{CC},\left\{a^{*}\right\} \succeq B$ This implies $A \succeq B$ by M and transitivity.

Proof of' Fact 3.2 First we show that for any $\succ \in \mathcal{P}_{C C}(X) \backslash\{\emptyset\}$ there oxusts $P^{0}(X) A \neq X$, such that $\succ_{A} \subset \succ$. This can he verified as follows Given $\succ \in P_{C C}(X)$, let $f: P^{\prime \prime}(X) \rightarrow P^{0}(., Y)$ be the mapping defined in (3.2). Observe. that by $\| 1$ and CC. $B \subseteq A$ implies $f(B) \subseteq f(A)$ (cf. [5, Lemma $2(\mathrm{~h}))]$ ). Also. one easily shows that for all $A \in P^{0}(X), f(f(A))=f(A)$ (cf. [5, Lemma 2(a) $)$ ) In particular, the sets of the forrn $f(A), A \in P^{0}(X)$, are precisely the fixfd points of the mapping $f$. Let $D \subseteq C$ be such that $C \succ_{f(A)} D$, i.e. $C \mathbb{E} f(A)$ and $\mathrm{D} \subseteq f(A)$. In particular, $f(D) \subseteq f(A)$. We will show that $C \succ D$ Assume to the contrary that $D^{-} C$. Then, by the definition of $f . C \subseteq f(D)$. hence $(; \subseteq f(A)$ However, this is false by assumption and therefore $(; \succ D)$ This shows that for each $A \in P^{0}(X), \succ_{f(A) \subseteq} \succ$. Finally, suppose that $\succ \neq \emptyset$. hence for some $(C, D) \in \mathcal{L}(X), C \succ D$. Then, $C \not \subset f(D)$, hence $f(D) \neq X$ This proves that if an element of $P_{C C}(X) \backslash\{\emptyset\}$ is minimal it is contained in $\mathcal{P}_{i c}^{*}(X) \backslash\{\emptyset\}$.

It remains to be shown that indeed every element of $\mathcal{P}_{C C}^{*}(X) \backslash\{\emptyset\}$ is muirnal. Hence. let $A, B \in P^{3}(X)$ such that $A \# X$ and $\succ_{A} \subset \succ_{B}$. By defi. nition of $\succ_{A}$, one has for all $r \notin A, A \cup\{x\} \succ_{A} A$. Hence, by assumption $A \mathrm{u}\{x\} \succ_{B} A$. which by definition of $\succ_{B}$ is only possible when $A=B$. (.'oweguently, $\succ_{A} \subset \succ_{B}$ implies $\mathrm{A}:=B$ which immediately implies minimality of each clement in $P_{C C}^{*}(X) \backslash\{\emptyset\}$

Proof of Theorem 3.1 Consider the family $\mathrm{A}=\left\{f(A): A \in P^{0}(X)\right\} \quad B y$ the proof of Fact 3.2, $\succ \|(A) \subseteq \succ$ for all $A \in P^{n}(X)$. Hence, it suffices to show that for all $(C, D) \in \mathbb{Y}(X), C \succ D$ implies $C \succ_{f(A)} D$ for some $A \in P^{(0)}(X)$. However, by the definition of $f: P^{0}(X) \rightarrow P^{0}(X), C \succ D$ implies $C: \not \subset f(D)$ Also, one has $D \subseteq f(D)$. Hence, by definition of $\succ_{f(D)}, C \succ_{f(D)} D$

Proof of Theorem 4.1 Necessity of $\operatorname{SM}\left(P_{\succ}\right)$ can easily be chrcked along the lines of Example 4.1 Sufficiency of $\operatorname{SM}\left(P_{>}\right)$is verified by considering the maximal chain representation (3.4). It is shown that for each maximal chain $C$ the 
corresponding preference ordering $P_{\mathcal{C}}$ satisfies $x P_{i \rightarrow} y \Rightarrow x P_{\mathcal{C}} y$, provided that $\succ$ satisfies condition $\mathrm{SM}\left(P_{\succeq}\right)$ Thus, let $\mathcal{C}=\left\{H_{1}, \ldots, H_{m}\right\}$ be a maximal chain of fixed points of the mapping $f$ defined in (3.2) such that

$$
H: \subseteq H_{2} \subseteq \ldots \subseteq H_{m}=X
$$

Let $P_{\mathcal{C}}$ denote the preference ordering on $\mathrm{X}$ corresponding to that maximal chain. Obviously, for all $z, w \in X$,

$$
z P_{\mathcal{C}} w \Leftrightarrow \text { for some } j \in\{1, \ldots, \mathrm{m}), z \notin H_{j} \text { and } w \in H_{j} .
$$

Now let $\mathrm{x}, \mathrm{y} \in \mathrm{X}$ be given such that $x P_{>}$, and let $j_{0}$ be the minimal index such that $\mathrm{x} \in H_{j_{0}}$. First, we show that $j_{0}>1$. Indeed, assume to the contrary that $\mathrm{x} \in H_{1}$, In this case, $H_{1} \succeq\{\mathrm{x}\}$ and $\{x\}^{-}\{\mathrm{s}, y\}$ hence using the fact that $f\left(H_{j}\right)=H_{\text {, }}$ one would obtain $y \in H_{1}$. This in turn implies $f(\{y\}) \subseteq H_{1}$ However, $\mathrm{x} \notin \mathrm{f}(\{y\})$, hence $f(\{y\})$ is a proper subset of $H_{1}$ which contradicts maximality of the chain $\mathcal{C}$. This proves $j_{\cup}>1$

Next, we show that $y \in H_{j_{0}-1}$. Again, assume to the contrary that $y \notin$ $H_{j_{0}-1}$. Then, $H_{j_{0}-1} \mathrm{u}\{y\}>H_{j_{0}-1}$. Let $H^{\prime}:=f\left(H_{j_{1-1}-1} \mathrm{u}\{y\}\right)$. Clearly, $H f^{\prime}$ is a proper superset of $H_{30-1}$. Also observe that $H_{j_{0}} \succeq\{x\}-\{x, y\}$ implies $y \in H_{j_{0}}$, and therefore, $H^{\prime} \subseteq H_{j_{0}}$. We now show that $x^{\prime} \notin H^{\prime}$. Indeed. by condition $\operatorname{SM}\left(P_{\succ}\right), H_{j_{0}-1} \cup\{y\} \succ H_{j_{0}-1}$ implies $H_{j_{0}-1} \cup\{y\} \cup\{x\} \succ H_{j_{0}-1} \cup\{y\}$, hence $x \notin \mathrm{H}^{\prime}$. But this implies that $H^{\prime}$ is a proper subset of $H_{j 0}$ which again contradicts maximality of the chain $\mathcal{C}$. Therefore one must have $y \in H_{j_{0}-1}$ From this one finally obtains $x P_{\mathcal{C}} y$ using (A.2).

Hence, $x P_{>} x \Rightarrow x P_{i} y$ for all $i$ if $\left\{P_{1}, \ldots, P_{n}\right\}$ is the representung family corresponding to the maximal chain representation of $\succ$. Conversely, it is obvious that $\left\{x P_{i} y\right.$ for all $\left.i \in\{1, \ldots, n\}\right] \Rightarrow x P_{\succ} y$.

Proof of Theorem 5.1 (Necessity of IIE) In order to verify necessity of IIE, suppose that $A \mathrm{U}\{\mathrm{s} y\} \sim A \cup\{x\}, A \mathrm{U}\{x, y\} \sim A \cup\{y\}$ and riot $x I_{z} y$. Let $\mathcal{R}=\left\{R_{1} . \quad \ldots R_{n}\right\}$ be a representing family for $\succeq$ that is closed under compromise Assume contrary to what LIE claims, that $A \cup\{x, y\} \succ A$. 'This implies that for sornc $\mathbf{j} . A \mathrm{u}\{x, \mathrm{y}\} \succ_{j} A$ where $\succeq_{j}=I U\left(R_{l}\right)$ Without loss of generality, suppose that $x R_{3} y$. Given this, one can conclude that $x P_{j} a$ for all $a \in A$. Since by assumption. $A \cup\{\mathrm{x}, \mathrm{y}\} \sim A \cup\{y\}$ one must have $z R_{j} x$ for some $z \in A \cup\{y\}$, hencc $y R, x$ and therefore $x I_{j} y$. Since not $x I_{\succ} y$ there must exist $\mathrm{k} \neq \mathbf{j}$ such that $y P_{k} x$, or the exists $l \neq \mathbf{j}$ such that $x P_{l} y$. In the first case, consider the convex-combination $\lambda u_{k}+(1-\lambda) u_{j}$, where $u_{k}, u_{\text {j }}$ represent $R_{k}, R_{j}$, respectivley, and let $R_{\lambda}$ denote the corresponding preference ordering in $\mathcal{R}$ For sufficiently small (but positive) $\lambda$ one obtains $y P_{\lambda} x$ and $y P_{\lambda} a$ for all $a \in \mathrm{A}$. However, this contradicts the assumption that $A \cup\{x, y\} \sim A \cup\{x\}$ In the second case, a symmetric argument can be applied in order to derive a contradiction to the assumption that $A \cup\{x, y\}-A \cup\{y\}$. Hence, in both cases one can conclude $A \cup(\mathrm{s}, y\} \sim A$ as required by IIF, The sufficiency part, 
of Theorem 51 is conveniently based upon Theorem 61 Hence, we prove that result first

Proof of Theorem 6.1 Necessity of liE is easily verified. The proof of the sufficiency part consists in showing that, given condition IIE, the representing family corresponding to the maximal chain representation of $\succeq$ is effectively linear. Let $\mathcal{C}=\left\{H_{1}, \ldots, H_{m_{2}}\right\}$ be a maximal chain of pairwise different fixed points of the mapping $\mathrm{f}$ defined in (3.2) such that $H_{m}=\mathrm{X}$ and $H_{j} \subset H_{j+1}$ for $j \in\{1, \ldots, \mathrm{m}-1)$. From (A.2) it is clear that the representing family corresponding to the maximal chain representation is effectively linear provided that (i) $\{\mathrm{v}, \mathrm{w}) \subseteq H_{1}$ implies $v I_{\succ} w$, and (ii) for all $\mathbf{j} \in\{\mathbf{1}, \ldots, \mathbf{m}-1\},\{v, \mathbf{w}) \subseteq H_{j+1} \backslash H_{j}$ implies $v I_{\succ} w$. In order to verify (I), suppose that $\{v, \mathrm{w}\} \subseteq H_{1}$ for $v \neq w$. By $\mathrm{CC}, f(\{v, w\}) \subseteq H_{1}$. Assume that not $v I_{\succ} w$, e $\{v, w\} \succ\{v\}$ or $\{v, w\} \succ\{w\}$. Without loss of generality. we ma, assume that $\{v, w\}>\{\mathrm{c}\}$. However, this would imply that $f(\{v\})$ is a. proper subset of $H_{1}$ which contradicts maximality of the chain. Hence, one must have $v l_{\succ} u$

Next we show (ii). Suppose that $\left.\left\{u^{\prime}, a^{\prime}\right\} \subseteq H_{3}+1\right\} H_{3}$ for $\#$ \# In particular. $H, \cup\{\mathrm{I}\}>H_{3}$, and $H, \cup\{u\}>H$, Consider $H^{\prime}=f(H, \cup\{L\})$ and $H^{\prime \prime}=$ $f(H, \cup\{u\}) \quad B y$ condition $\left(\subseteq . E l^{1} . H^{\prime \prime} \subseteq H_{j+1}\right.$, hence by maximality of the chain $C^{\prime} . H^{\prime}=H^{\prime \prime}=H_{j+1}$ This implies $H_{j} \cup\{v, w\} \sim H, \cup\{v\}$ and $H_{j} \cup\{v, w\} \sim$ $H_{3} \cup\{w\}$ Now assume that not $v l_{\succ} w$ Then. IIE would imply $H_{j} \cup\{v, w\} \sim H_{\text {, }}$ which contradicts the fact that $\mathrm{H}$, is a fixed point of $f$ Hence, $I>w$

Proof of Theorem 5.1 (Sufficiency of IIE) Let $\succeq \in R_{C} \cdot(X)$ satisfy $11 \mathrm{H}$

let $\left\{R_{1}, \quad, R_{n}\right\}$ be a representing family for $\succeq$ that is effectively linear according to Theorem 61 . The following proof is based upon the construction in $[7, \mathrm{Th} 6]$ Fix such that $0<t<1 / n$, and define for each $\imath=1, n$ and all $x \in$ $X . u_{i}(x)=\epsilon^{\#\left\{z \in X: R_{1} x\right\}}$ Obviously, for all $\imath=1, \ldots, n, u$, represents $R$, It will be shown that the convex hull $\mathcal{U}$ of $\left\{u_{1} . \quad . u_{n_{2}}\right\}$ constitutes a representung family for $\succ$ as well Let $(A, B) \in Z(X)$ Obviously. $\max _{x \in A} u(x)>\max _{x \in B} u(x)$ for all $u \in \mathrm{I} 4$ implies $A \succeq B$ The converse implication is shown by a contradiction argument Hence suppose that $A>B$. I $\mathrm{p}$ for all $i=1 \ldots n$.

$$
\max _{x \in A} u_{i}(x) \geq \max _{x \in B} u_{i}(x)
$$

while for some $u \in U$ and some b $\in B$.

$$
u(b)>u(x) \text { for all } x \in A \text {. }
$$

Let $u=\sum_{i=1}^{n} \lambda_{2} u_{i}$. For all $i=1 \ldots n$. define $\lambda_{i}^{*}:=\lambda_{i} u_{2}(b) /\left(\sum_{j} \lambda_{j} u_{j}(b)\right)$. By $(A .4)$

$$
1>\sum_{i=1}^{n} \lambda_{i} \frac{u_{i}(r)}{u_{i}(b)} \text { for all } x \in A
$$

For all 7 . let $x_{i}^{*} \in \arg \max _{s \in A} u_{\imath}(x)$ We now show that. for all $\imath, x_{i}^{*} P_{i} b$ Indeed. by $(\lambda: 3)$ one has $x_{2}^{*} R_{i} b$ On the other hand, $x_{k}^{*} l_{k} b$ for some $k$ would imply by 
effective linearity! $x_{i}^{*} I_{i} b$ for all $i$, which is not possible by (A.4). Consequently, for all $i$,

$$
\frac{u_{i}\left(x_{i}^{*}\right)}{u_{i}(b)} \geq \frac{1}{\epsilon}>n
$$

Since, $u_{i}(z)$ is non-negative for all $\mathrm{i}$ and all $z \in \mathrm{X}$, (A.5) and (A.6) together imply that for all $\mathrm{i}, \lambda_{i}^{*}<1 / n$. Howevrr, this contradicts the fact that the $\lambda_{i}^{*}$ add up to 1 .

Proof of Fact 7.1 Given $(\mathrm{A}, B) \in \Sigma(X)$, it is clear that $(A \backslash B) \cap E(A) \neq 0$ implies $\mathrm{A} \succ \mathrm{B}$. Conversely, let $\mathrm{B} \subseteq \mathrm{A}$ and suppose that for all $x \in A \backslash B$, $A \sim A \backslash\{\mathrm{x})$. Then, by succesive application of IlE, A $-B$. This proves (i).

In order to verify (ii), assume that for some $A \in P^{U}(X)$ with \#A $\geq 2$, $E(A)=\emptyset$. Hence, for some $\imath \neq w,\{v, w\} \subseteq \mathrm{A}$ and $A-A \backslash\{x\}$ for every $x \in \mathrm{A}$ Succesive application of IIE implies $\mathrm{A}^{-}\{v\}$ and $A^{-}\{u\}$. Hence by condition $\mathrm{M},\{v, w\} \sim\left\{v \mid\right.$ and $\{v, w\}{ }^{-}\{u\}$ which contradicts the assumptions. Thus $E(A) \# 0$ for all $A \in P^{0}(X)$

Proof of Fact 7.2 Parts (i) (iv) are easily verified. Hence, it suffices to show that satisfies condition IIE. Clearly, $A \cup\{x\} \cup\{\mathrm{y}\}-\mathrm{A} \cup\{\mathrm{r}\}$ and $A \cup\{x\} \cup\{\mathrm{y}\} \sim A \mathrm{U}\{y\} \operatorname{imply} y \notin G(A U\{\mathrm{r}\} \mathrm{U}\{\mathrm{y}\})$ and $x \notin C(A \mathrm{u}\{\mathrm{x}) \cup\{y\})$, respectively. However, this implies by $\operatorname{SD}(G), A \cup\{x, y\} \sim A$

Proof of Theorem 7.1 Given $\mathrm{SD}(G)$, the equivalence between $\mathrm{CC}$ and $(\alpha)$ is easily verified. In order to deduce $(A i z)$ from negative transitivity, observe that for $\mathrm{B} \subseteq \mathrm{A}, G(A) \subseteq B$ implies $A \sim B$. Sow suppose that $\mathrm{x} \in G(B)$. i.e. $B \succ \mathrm{B} \backslash\{x\}$. By negative transitivity, $A \succ \mathrm{B} \backslash\{\mathrm{x}\}$, hence by $\mathrm{SD}$,

$$
[(A \backslash B) \cup\{x\}] \cap C(A) \neq 0
$$

Given $G(A) \subseteq B$ this immediately implies $x \in G(A)$. Finally, the last statement in Theorem 7.1 follows from the observation that under SD, $(\alpha)$ and (Aiz), for all $B \subseteq A, A \sim B \Leftrightarrow C(A)=G(B)$

Proof of Corollary 7.1 Vecessity of $(\alpha)$ and (Aiz) is obvious In order to show their sufficiency, define a binary relation $\succeq$ in $\Sigma(X)$ by conditions $\mathrm{M}$ and $\operatorname{SD}(G)$. By Theorem 7.1, $\succeq$ is an clement of $\mathcal{R}_{C C}(X)$. By Fact $72(\mathrm{v}), \succeq$ satisfies IIE. Furthermore, by Fact 72 (iii), any rigid indifference 1 s trivial. Hence, by Theorem 6.1 there exists a representing family $\left\{P_{1}, \ldots, P_{n}\right\}$ for $\succeq$ that consists of linear orderings. It can be verified that $\left\{P_{1} . ._{n}\right\}$ rationalizes $G$ in the sense of Corollary $\mathrm{T} .1$

\section{References}

[1] Aizerman, M.A. and Malishevski, A.V. (1981), "ieneral Theory of 
Best Variants Choice Some Aspects," IEEE Trans. Automat Control 26 10301040

[2] Bossert, W. Pattanalk, P.K. and Xu, Y. (1994), Ranking Opportunity Sets: An Axiomatic Approach," J. Econ. Theory 63, 326-345

[3] Gravel, N (1994), "Can a Flanking of Opportunity Sets Attach an Intrinsic Importance to Freedom of Choice," Amer. Econ. Rev. Pap. Proc. $84,454-458$

[4] Jones, P and Sugden, R. (1982), "Evaluating Choice," Int. Rev. Law and Écon. 2, 47-65.

[5] Krf.ps. D M (1979), "A Representation Theorem for 'Preference for Flexibility" "Econometrica 47, 565577

[6] Houlin, H (1985), "Choice Functions over a Finite Set: A Summary," soc Choue Welfare 2. 147-160.

[7] CEhring $\mathrm{K}$ (1996), "Rational Choice and Revealed P'reference without Binarmess." Soc. Choice Welfare, fortheoming.

[8] Nerring, $K$ and Peppe, $C$ (1996), "Continuous Extensions ot' an Order on a Set to the Power Set," J. Econ. Theory 68, 456-479.

[9] PATTANik, P.K and Xu, Y (1990): "On Ranking Opportunity Sets in Terms of Freedom of Choice." Recit. Econ. Louvain 56(3-4), 383-390.

[10] Pattanak. P.K and Xu, Y. (1995), "On Preference and Freedom mimeographed.

[11] Puppe, ( (1995). "Freedom of Choice and Rational Decisions," Soc. Chote Welfare 12. 137-153

[12] Plppe. ( (1996a) "An Axiomatic Approach to Preference for Freedom of Choice", J. Econ. Theory 68, 174-199.

[13] SEN $1 \mathrm{~K}(1970)$ Collectue Chorce and Socral Welfare San Francisco Holden-Day

[14] SEN, A.K (1988). "Freedom of (hoice: Concept and Content," Eur. Ecom. Rev. 32, 269-294

[10] Sex. A K. (1991). "Wolfare, Preference and Freedom." J. Econometrics $50.15-29$. 


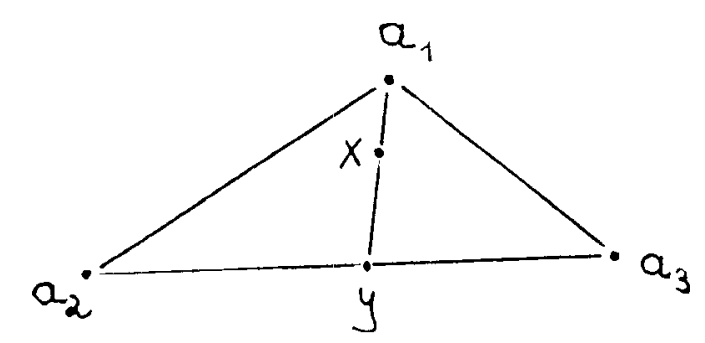

Figure 1

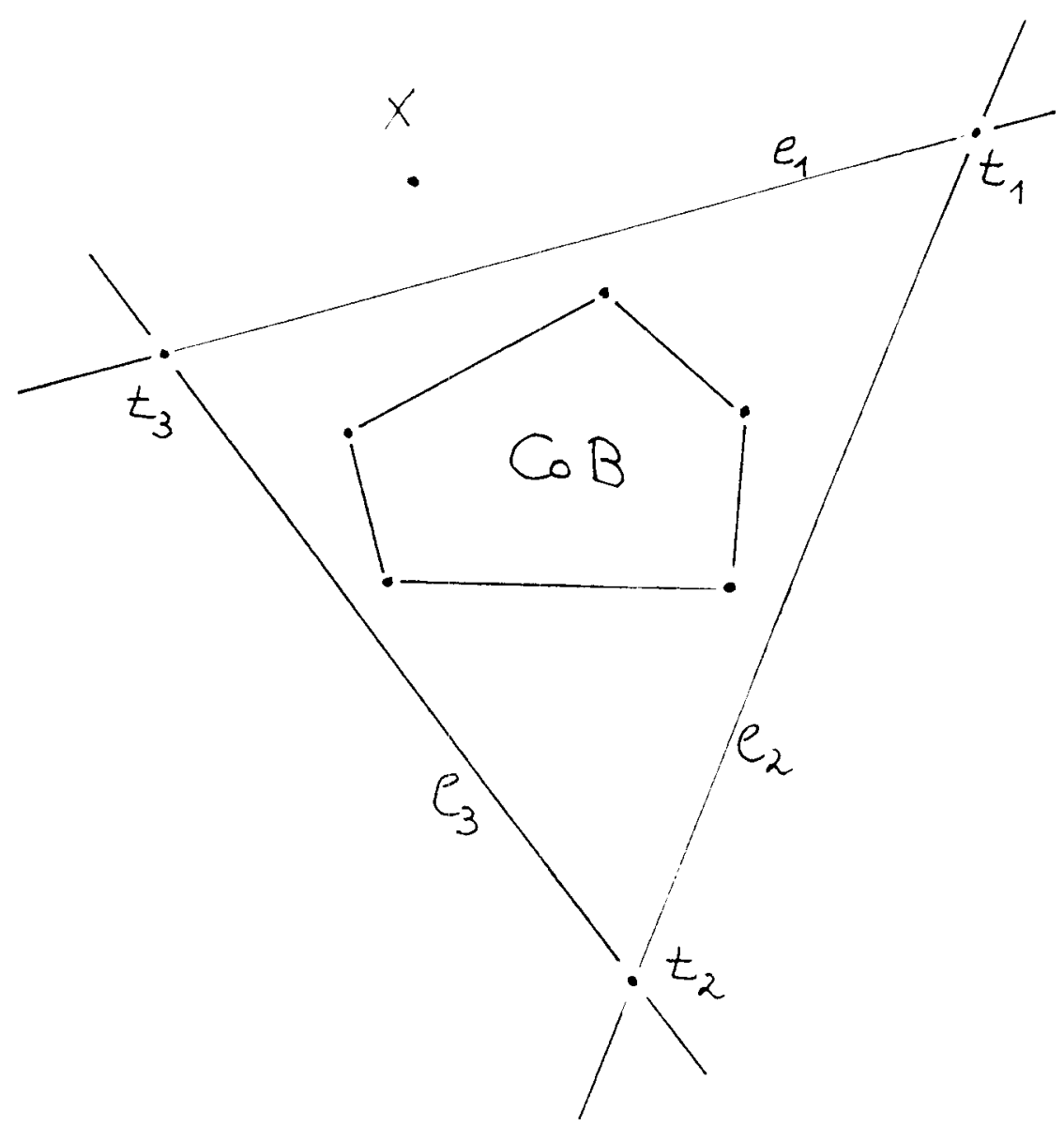

Figure 2 